\title{
Statistical distribution of thermal vacancies close to the melting point
}

\author{
María José Pozq* and Sergio Davis ${ }^{\dagger}$ \\ Grupo de Nanomateriales, Departamento de Física, \\ Facultad de Ciencias, Universidad de Chile, Casilla 653, Santiago, Chile \\ Joaquín Peralta \\ Departamento de Ciencias Físicas, Facultad de Ciencias Exactas, Universidad Andrés Bello, Santiago, Chil $\bigoplus^{\mp}$
}

(Dated: June 15, 2021)

\begin{abstract}
A detailed description of the statistical distribution of thermal vacancies near the melting point is presented, using copper as an example. As the temperature is increased, the average number of thermal vacancies generated by atoms migrating to neighboring sites also increase, according to Arrhenius' law. We present for the first time a model for the distribution of thermal vacancies, which according to our results follow a Gamma distribution. All the simulations are carried out by classical molecular dynamics and the recognition of vacancies is achieved via a recently developed algorithm. Our results could be useful in the further development of a theory explaining the mechanism of homogeneous melting, which seems to be mediated (at least in part) by the accumulation of thermal vacancies near the melting point.
\end{abstract}

\section{INTRODUCTION}

Understanding the production of thermal vacancies due to atomic migration near the melting temperature $T_{m}$ should provide relevant information on the melting process itself. Recent studies involving computer simulation ${ }^{115}$ have connected the catastrophic collapse of the crystal in homogeneous melting to a collective (ring-like) movement of atoms due to thermally produced vacancies. In order to construct a quantitative model, however, there is a key piece missing: the statistical distribution of thermal vacancies at a given temperature $T$.

For temperatures close to $T_{m}$, the expected concentration of vacancies is between $10^{-3}$ and $10^{-4}$ for metals ${ }^{6}$. However, from this point on it is naturally expected (and indeed true) that the number of thermal vacancies increases. Another common assumption is that, at a fixed temperature $T$, the concentration of vacancies $f_{v}=n_{v} / N$ (where $n_{v}$ is the number of vacancies and $N$ the total number of atoms) is normally distributed around an average value $\left\langle f_{v}\right\rangle$ which follows Arrhenius' law,

$$
\left\langle f_{v}\right\rangle_{T}=\exp \left(-E_{v} / k_{B} T\right)
$$

where $E_{v}$ is the free energy of formation of thermal vacancies ${ }^{6}$.

In this work we provide evidence from atomistic computer simulations supporting a Gamma model for the concentration of thermal vacancies in copper near $T_{m}$ (but below $T_{L S}$ ).

The work is organized as follows. Section II shows a detailed description of the molecular dynamics and vacancy recognition procedures. Section III shows the details of the inference process employed for the statistical comparison of the Gamma and normal models for vacancy distribution. Section IV] comments on the scope and implications of our results.

\section{SIMULATION TECHNIQUES}

The simulations were performed using an FCC copper structure composed of 1372 atoms ( $7 \times 7 \times 7$ unit cells), using a lattice parameter of $a=3.61 \AA$ in a cubic cell of length $L=25.27 \AA$, as shown in Fig. 11. The lattice parameter corresponds to room pressure copper.

Classical molecular dynamics (MD) simulations were performed in the microcanonical ensemble (i.e., with fixed $N, V$ and $E$ ) using the LPMD software package $e^{7}$. We described the interatomic interactions in copper by the Sutton-Chen potential with the usual parameterization ${ }^{8}$.

Simulations at four different initial temperatures, 2300 $\mathrm{K}, 2500 \mathrm{~K}, 2600 \mathrm{~K}$, and $2700 \mathrm{~K}$, were performed for 50 ps each, with a timestep $\Delta \mathrm{t}=1 \mathrm{fs}$. In all cases the initial temperature $T_{0}$ was set to be about twice the target temperature $T$, and the ideal crystalline structure is used as the initial positions. In this manner equilibration is achieved without the use of thermostats which could distort the natural dynamics of the system. This is the same microcanonical approach used in the Z-method 9 . The temperatures were chosen close to the experimental melting point of copper, $T_{m} \sim 1360 \mathrm{~K}$, in order to have a non-zero probability of observing thermal vacancies given the size of the system.

We computed the radial distribution function $g(r)$ for all the temperatures in order to check that we indeed have a solid structure in all cases. Figure 2 shows the $g(r)$ for the case of $T=1400 \mathrm{~K}$, all the other temperatures being almost identical. In this figure we can see that the nearest-neighbors peak is located around $r=2.5 \AA$, which gives the approximate radius of a spherical vacancy to be close to $1.25 \AA$.

To determine the number of vacancies generated during the simulations, the Search-and-Fill algorithm ${ }^{[10}$ was used. This technique generates virtual spheres in the simulation cell (of radius $R_{0}$ ) and tries to place them with minimum overlap with the atoms. Every site where a 


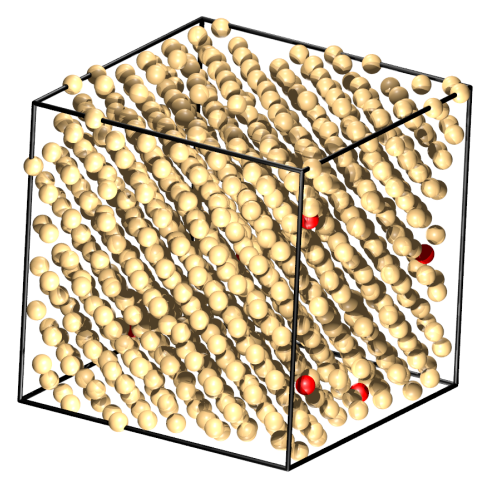

FIG. 1. Copper structure snapshot from the MD simulation at $T=1400 \mathrm{~K}$. The structure has 1372 atoms, with an initial FCC crystalline structure. The red spheres represent thermal vacancies located with the Search-and-Fill algorithm.

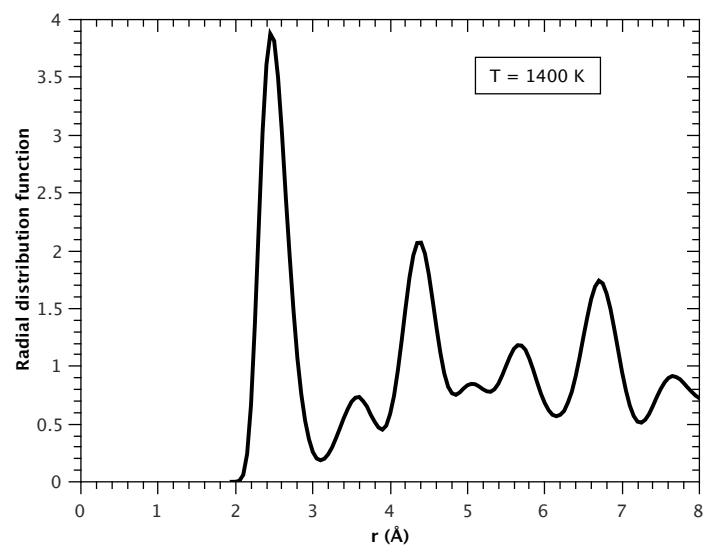

FIG. 2. Pair distribution function for copper at $T=1400 \mathrm{~K}$. It shows the structure is still an FCC solid, with the usual broadening of the peaks due to temperature.

virtual sphere can fit with an overlap below a threshold $\Omega$ is identified as a vacancy and the site is filled (i.e., the site is not considered empty for the purposes of locating the next vacancy). In the particular case of copper we used the values of $R_{0}=1.275 \AA$ and the threshold overlap parameter $\Omega=0.4$.

The average results obtained from the vacancy recogni-

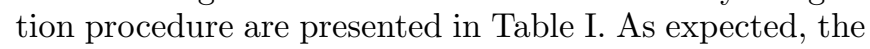
concentration of vacancies increases with temperature.

\section{VACANCY DISTRIBUTION}

To determine how the vacancies are distributed in the sample we evaluate the number of vacancies in each time

\begin{tabular}{cc}
\hline $\mathrm{T}(\mathrm{K})$ & $\left\langle f_{v}\right\rangle\left(10^{-3}\right)$ \\
\hline 1200 & 2.06195 \\
1300 & 3.22012 \\
1350 & 3.97303 \\
1400 & 4.62318 \\
\hline
\end{tabular}

TABLE I. Values of the average concentration of thermal vacancies for several temperatures.
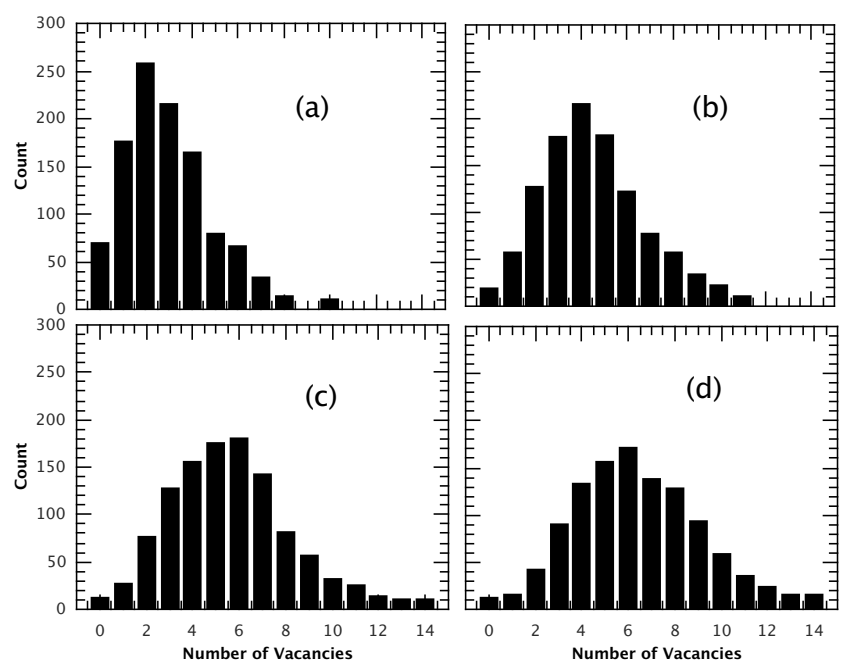

FIG. 3. Vacancy distribution corresponding to each temperature. The histogram represents the frequency of each number of vacancies present in a sample of 1372 atoms.

step during the simulation. The results of this vacancy count are organized in a histogram. These histograms for four different temperatures are displayed in Fig. 3.

We propose two models for the probability distribution of vacancies, a Gaussian distribution,

$$
P\left(f_{v} \mid \mu, \sigma\right)=\frac{1}{\sqrt{2 \pi} \sigma} \exp \left(-\frac{1}{2 \sigma^{2}}\left(f_{v}-\mu\right)^{2}\right)
$$

where $\mu$ and $\sigma^{2}$ are the mean and variance of $f_{v}$, respectively, and a Gamma distribution, given by

$$
P\left(f_{v} \mid k, \theta\right)=\frac{1}{\Gamma(k) \theta^{k}} f_{v}^{k-1} \exp \left(-f_{v} / \theta\right)
$$

where $k$ and $\theta$ are its shape and scale parameters, respectively. In both cases the parameters of the distributions are functions of $T$. In order to compare both models using our simulated data for each temperature, the Bayesian Information Criterion (BIC) $)^{11}$, defined as

$$
\mathrm{BIC}=-2 \ln L\left(\hat{\lambda}_{\mathbf{0}}\right)+n_{p} \ln N,
$$

has been used, where $L(\lambda)$ is the likelihood function for the model, $\lambda_{\mathbf{0}}$ are the most probable parameters according to the maximum likelihood method, $n_{p}$ is the number 


\begin{tabular}{ccc}
\hline $\mathrm{T}(\mathrm{K})$ & BIC for Gamma & BIC for normal \\
\hline 1200 & 3852.5 & 3936.1 \\
1300 & 4273.7 & 4302.6 \\
1350 & 4475.4 & 4486.1 \\
1400 & 4695.9 & 4725.2 \\
\hline
\end{tabular}

TABLE II. Values of the Bayesian Information Criterion (BIC) for the Gamma and normal models applied to the concentration of thermal vacancies for several temperatures. In all cases the Gamma model is selected (has lower BIC) over the normal.

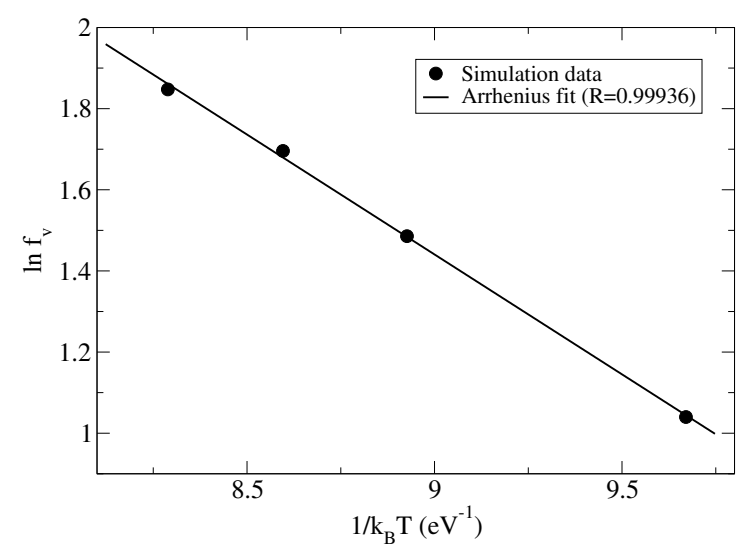

FIG. 4. Arrhenius law for the average concentration of vacancies.

of parameters in the model and $N$ is the number of data points. In this method, the lower the value of BIC, the better (the model gives a better fit to the data). In our case, the second term is the same for both models, and so the comparison reduces to a maximum likelihood ratio. The results for each temperature are displayed in Table II]

For all temperatures, the Gamma model is to be preferred over the normal model, and in all cases the difference in BIC is larger than 10 points, giving for each temperature odds in favor of the Gamma model higher than 140:1. We therefore conclude that the evidence in favor of the Gamma model is statistically conclusive for our data (see for instance Raftery ${ }^{12}$ for the statistical significance of Bayes factors and BIC differences).

The Arrhenius law (Eq. 1) for our calculated averages is displayed in Fig. 44 which corresponds to an activation energy $E_{v} \sim 0.59112 \mathrm{eV}$, lower than previous experimental results on intrinsic vacancies ${ }^{13 \mid 14}$ (reporting values around $1.0 \mathrm{eV}$ ).

This is to be expected, as the formation of an intrinsic vacancy is more costly, due to it involving the removal of an atom from the surface, while the thermal vacancy is actually a vacancy-interstitial pair, and involves just a local rearrangement of atomic overlap.

With the obtained value of $E_{v}$, the concentration of vacancies at the experimental melting point is $f_{v}\left(T_{m}\right) \sim$ $4 \times 10^{-3}$, in agreement with known values for metals ${ }^{6}$.

\section{CONCLUDING REMARKS}

The formation of thermal vacancies is a stochastic phenomenon which, however, seems to follow a well defined statistical distribution. We provide evidence supporting a Gamma distribution with long tails instead of the more common normal distribution, which increases the probability of larger concentrations at a given mean (extreme events). This has implications for the modelling of the homogeneous melting process which is dependent on the formation of thermal vacancies and their mobility, as the normal model would underestimate the probability of a critical vacancy concentration. The procedure employed in this work is capable of determining the free energy of formation of vacancies by performing molecular dynamics simulations or even Monte Carlo simulations.

\section{ACKNOWLEDGEMENTS}

SD \& JP gratefully acknowledge funding from FONDECYT grant 1140514.
* mariaj.pozo@gmail.com

$\dagger$ http://www.gnm.cl/ sdavis sdavis@gnm.cl

$\ddagger$ joaquin.peralta@unab.cl

1 F. Delogu, J. Phys. Chem. B 109, 15291 (2005).

2 X.-M. Bai and M. Li, Physical Review B 77, 134109 (2008).

3 L. C. Gallington and A. Bongiorno, The Journal of Chemical Physics 132, 174707 (2010).

4 S. Davis, A. B. Belonoshko, B. Johansson, and A. Rosengren, Physical Review B 84, 064102 (2011).

5 H. Zhang, M. Khalkhali, Q. Liu, and J. Douglas, J. Chem. Phys. 138, 12A538 (2013).

6 M. Hillert, Phase equilibria, Phase Diagrams and Phase Transformations (Cambridge University Press, 2007).
7 S. Davis, C. Loyola, F. González, and J. Peralta, Comp. Phys. Comm. 181, 2126 (2010).

8 A. P. Sutton and J. Chen, Phil. Mag. Lett. 61, 139 (1990).

9 A. B. Belonoshko, N. V. Skorodumova, A. Rosengren, and B. Johansson, Phys. Rev. B 73, 012201 (2006).

10 S. M. Davis, A. B. Belonoshko, and B. Johansson, Comp. Phys. Comm. 182, 1105 (2011).

11 G. E. Schwarz, Annals of Statistics 6, 461 (1978).

12 A. E. Raftery, Sociological Methodology 25, 111 (1995).

13 R. O. Simmons and R. W. Balluffi, Phys. Rev. 129, 1533 (1963).

14 W. Triftshäuser and J. D. McGervey, Applied Physics 6, 177 (1975). 